\title{
DUALITY FOR POWERSET COALGEBRAS
}

\author{
GURAM BEZHANISHVILI $^{a}$, LUCA CARAI $^{b}$, AND PATRICK J. MORANDI $^{a}$ \\ ${ }^{a}$ New Mexico State University \\ e-mail address: \{guram,pmorandi\}@nmsu.edu \\ ${ }^{b}$ Università degli Studi di Salerno \\ e-mail address: lcarai@unisa.it
}

\begin{abstract}
Let CABA be the category of complete atomic boolean algebras and complete boolean homomorphisms, and let CSL be the category of complete meet-semilattices and complete meet-homomorphisms. We show that the forgetful functor from CABA to CSL has a left adjoint. This allows us to describe an endofunctor $\mathcal{H}$ on CABA such that the category $\operatorname{Alg}(\mathcal{H})$ of algebras for $\mathcal{H}$ is dually equivalent to the category $\operatorname{Coalg}(\mathcal{P})$ of coalgebras for the powerset endofunctor $\mathcal{P}$ on Set. As a consequence, we derive Thomason duality from Tarski duality, thus paralleling how Jónsson-Tarski duality was derived from Stone duality in [Abr88, KKV04].
\end{abstract}

\section{INTRODUCTION}

It is a classic result in modal logic, known as Jónsson-Tarski duality, that the category MA of modal algebras is dually equivalent to the category DFr of descriptive frames. This result can be traced back to the work of Jónsson-Tarski [JT51], Halmos [Hal56], and Kripke [Kri63]. In the modern form it was proved by Esakia [Esa74] and Goldblatt [Gol76]. ${ }^{1}$

Jónsson-Tarski duality is a generalization of the celebrated Stone duality between the category BA of boolean algebras and the category Stone of Stone spaces. It was observed by Abramsky [Abr88] and Kupke, Kurz, and Venema [KKV04] that Jónsson-Tarski duality can be proved by lifting Stone duality using algebra/coalgebra methods. This can be done by utilizing the classic Vietoris construction (see, e.g., [Joh82, Ch. III.4]). Indeed, associating with each Stone space $X$ its Vietoris space $\mathcal{V}(X)$ gives rise to an endofunctor $\mathcal{V}:$ Stone $\rightarrow$ Stone such that DFr is isomorphic to the category Coalg $(\mathcal{V})$ of coalgebras for $\mathcal{V}$. Let $S L$ be the category of meet-semilattices with top. Then the forgetful functor $\mathcal{U}: \mathrm{BA} \rightarrow \mathrm{SL}$ has a left adjoint $\mathcal{L}: \mathrm{SL} \rightarrow \mathrm{BA}$. Letting $\mathcal{K}=\mathcal{L U}$ gives an endofunctor $\mathcal{K}: \mathrm{BA} \rightarrow \mathrm{BA}$ such that MA is isomorphic to the category $\operatorname{Alg}(\mathcal{K})$ of algebras for $\mathcal{K}$. Moreover, the following diagram commutes up to natural isomorphism, yielding that Stone duality lifts to a dual

\footnotetext{
Key words and phrases: Modal logic, coalgebra, Jónsson-Tarski duality, Thomason duality.

${ }^{1}$ We point out that Esakia phrased it for the subcategory of descriptive frames where the relation $R$ is reflexive and transitive. Consequently, he worked with the subcategory of modal algebras consisting of closure algebras of McKinsey and Tarski [MT44].
} 
equivalence between $\operatorname{Alg}(\mathcal{K})$ and $\operatorname{Coalg}(\mathcal{V})$. This provides an alternate proof of Jónsson-Tarski duality.

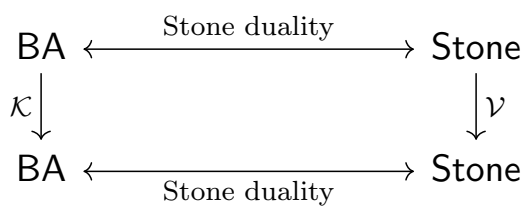

Descriptive frames can be thought of as Kripke frames $(X, R)$ equipped with a Stone topology compatible with the relation $R$. In [Tho75] Thomason proved a "discrete version" of Jónsson-Tarski duality, establishing that the category KFr of Kripke frames is dually equivalent to the category CAMA of complete atomic modal algebras whose modal operator is completely multiplicative (see Sections 2 and 3 for all the undefined notions).

The same way Jónsson-Tarski duality generalizes Stone duality, Thomason duality generalizes Tarski duality between the category CABA of complete atomic boolean algebras and the category Set of sets. It is natural to try to obtain Thomason duality from Tarski duality using algebra/coalgebra methods in the same vein Jónsson-Tarski duality was obtained from Stone duality in [Abr88, KKV04]. Surprisingly, such an approach has not yet been undertaken. Our aim is to fill in this gap.

For this purpose, it is natural to replace the Vietoris endofunctor $\mathcal{V}$ on Stone with the powerset endofunctor $\mathcal{P}$ on Set. It is known (see, e.g., [Ven07, Sec. 9]) that KFr is isomorphic to Coalg $(\mathcal{P})$. Thus, the key is to construct an endofunctor $\mathcal{H}$ on CABA that is an analogue of the endofunctor $\mathcal{K}: \mathrm{BA} \rightarrow \mathrm{BA}$. We recall that $\mathcal{K}=\mathcal{L U}$ where $\mathcal{L}: \mathrm{SL} \rightarrow \mathrm{BA}$ is left adjoint to the forgetful functor $\mathcal{U}: \mathrm{BA} \rightarrow \mathrm{SL}$. A natural analogue of $\mathrm{SL}$ in the complete case is the category CSL of complete meet-semilattices. Our main contribution is to show that the forgetful functor $\mathcal{U}: C A B A \rightarrow C S L$ has a left adjoint $\mathcal{L}: C S L \rightarrow C A B A$. We then define $\mathcal{H}: \mathrm{CABA} \rightarrow \mathrm{CABA}$ as the composition $\mathcal{H}=\mathcal{L U}$, and prove that $\operatorname{Alg}(\mathcal{H})$ is dually equivalent to $\operatorname{Coalg}(\mathcal{P})$. Since CAMA is isomorphic to $\operatorname{Alg}(\mathcal{H})$ and $\operatorname{Coalg}(\mathcal{P})$ is isomorphic to $\mathrm{KFr}$, Thomason duality follows.

The paper is organized as follows. In Section 2 we recall Jónsson-Tarski duality and how it can be obtained by lifting Stone duality. For this we need to work with the left adjoint $\mathcal{L}: \mathrm{SL} \rightarrow \mathrm{BA}$ of the forgetful functor $\mathcal{U}: \mathrm{BA} \rightarrow \mathrm{SL}$. The standard approach to constructing $\mathcal{L}$ is to take the free boolean algebra over the underlying set of a meet-semilattice with top and quotient it by the relations defining a modal operator $\square$ (see [KKV04, Prop. 3.12]). As we will show in Theorem 2.5, $\mathcal{L}$ can alternatively be constructed by utilizing Pontryagin duality for semilattices [HMS74]. Let StoneSL be the category of topological meet-semilattices, where the topology is a Stone topology. Then SL is dually equivalent to StoneSL (see [HMS74, Thm. 3.9] or [Joh82, p. 251]). We will show that $\mathcal{L}$ can be constructed by taking the boolean algebra of clopen subsets of the Pontryagin dual $M^{*}:=\operatorname{hom}_{\mathrm{SL}}(M, 2)$ of $M \in \mathrm{SL}$. This shows that $\mathcal{L}: \mathrm{SL} \rightarrow \mathrm{BA}$ can be constructed either purely algebraically, by utilizing the existence of free algebras in BA, or using duality, as the boolean algebra of clopens of the Pontryagin dual of a meet-semilattice with top.

In Section 3 we show that the same approach applies to the forgetful functor $\mathcal{U}: C A B A \rightarrow$ $\mathrm{CSL}$. Its left adjoint $\mathcal{L}: \mathrm{CSL} \rightarrow \mathrm{CABA}$ can be constructed by taking the free object in CABA over the underlying set of a complete meet-semilattice and then taking the quotient of it by the relations defining a completely multiplicative modal operator $\square$. We point out that care is needed in constructing the free object in CABA since it is well known that free objects do not exist in the category of complete boolean algebras (see [Gai64, Hal64]). Nevertheless, free 
objects in CABA do exist. This can be seen by observing that the Eilenberg-Moore algebras of the double contravariant powerset monad are exactly the objects of CABA [Tay02], and that categories of algebras for monads have free objects [AHS06, Prop. 20.7(2)]. A more concrete construction of free objects in CABA can be given by utilizing the theory of canonical extensions of Jónsson and Tarski [JT51]. Indeed, in Theorem 3.4 we will prove that the free object in CABA over a set $X$ is the canonical extension $F^{\sigma}$ of the free boolean algebra $F$ over $X$. We then quotient $F^{\sigma}$ by the complete congruence generated by the relations defining a completely multiplicative modal operator, yielding the desired $\mathcal{L}: \mathrm{CSL} \rightarrow \mathrm{CABA}$ (see Theorem 3.7).

An alternate construction of $\mathcal{L}: \mathrm{CSL} \rightarrow \mathrm{CABA}$ that parallels the alternate construction of $\mathcal{L}: \mathrm{SL} \rightarrow \mathrm{BA}$ can be given by taking the powerset of $\operatorname{hom}_{\mathrm{CSL}}(M, 2)$ for each $M \in \mathrm{CSL}$. Since $\operatorname{hom}_{\mathrm{CSL}}(M, 2)$ is isomorphic to the order-dual of $M$, this amounts to taking the powerset of $M .^{2}$ Therefore, we again obtain that the left adjoint can be constructed either purely algebraically, utilizing that free objects exist in CABA, or else using the powerset construction. Thus, we arrive at the following diagram, which parallels the constructions of the left adjoints $\mathcal{L}: \mathrm{SL} \rightarrow \mathrm{BA}$ and $\mathcal{L}: \mathrm{CSL} \rightarrow \mathrm{CABA}:$

\begin{tabular}{|l|l|l|}
\hline Forgetful functor & Left adjoint & Location \\
\hline $\mathcal{U}: \mathrm{BA} \rightarrow \mathrm{SL}$ & $\begin{array}{l}\mathcal{L}: \mathrm{SL} \rightarrow \mathrm{BA} \\
\text { algebraic construction: } \operatorname{Free}_{\mathrm{BA}}(M) / \equiv \\
\text { dual construction: } \mathfrak{c l o p}(\operatorname{hom} \mathrm{SL}(M, 2))\end{array}$ & $\begin{array}{l}{[\text { KKV04] }} \\
\text { Theorem } 2.5\end{array}$ \\
\hline $\mathcal{U}: \mathrm{CABA} \rightarrow \mathrm{CSL}$ & $\begin{array}{l}\mathcal{L}: \mathrm{CSL} \rightarrow \mathrm{CABA} \\
\text { algebraic construction: } \operatorname{Free}_{\mathrm{CABA}}(M) / \equiv \\
\text { dual construction: } \wp\left(\operatorname{hom}_{\mathrm{CSL}}(M, 2)\right) \cong \wp(M)\end{array}$ & $\begin{array}{l}\text { Theorem } 3.7 \\
\text { Theorem } 3.9\end{array}$ \\
\hline
\end{tabular}

In Section 4 we define the endofunctor $\mathcal{H}:$ CABA $\rightarrow$ CABA as the composition $\mathcal{H}=\mathcal{L U}$. In Theorem 4.3 we prove that the following diagram commutes (up to natural isomorphism).

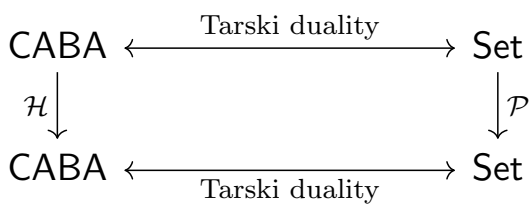

This paves the way towards proving that the category $\operatorname{Alg}(\mathcal{H})$ of algebras for $\mathcal{H}$ is dually equivalent to the category $\operatorname{Coalg}(\mathcal{P})$ of coalgebras for $\mathcal{P}$ (see Theorem 4.10).

It is well known that Jónsson-Tarski and Thomason dualities are connected through the canonical extension and forgetful functors $(-)^{\sigma}:$ MA $\rightarrow$ CAMA and $\mathcal{U}:$ DFr $\rightarrow \mathrm{KFr}$, making the following diagram commutative.

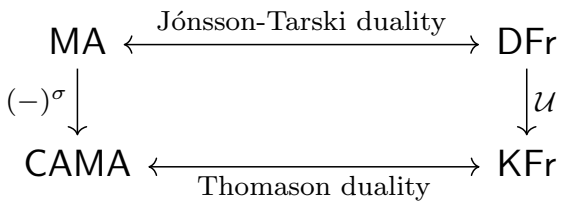

We conclude the paper by Remark 4.13 in which we show that there are analogous canonical extension and forgetful functors $(-)^{\sigma}: \operatorname{Alg}(\mathcal{K}) \rightarrow \operatorname{Alg}(\mathcal{H})$ and $\mathcal{U}: \operatorname{Coalg}(\mathcal{V}) \rightarrow \operatorname{Coalg}(\mathcal{P})$ that make a similar diagram commutative.

\footnotetext{
${ }^{2}$ We thank one of the referees for suggesting this approach.
} 


\section{CoAlgebraic approach to Jónsson-TARski DuAlity}

In this section we give a brief account of Jónsson-Tarski duality, and provide a construction of the left adjoint $\mathcal{L}: \mathrm{SL} \rightarrow \mathrm{BA}$ of the forgetful functor $\mathcal{U}: \mathrm{BA} \rightarrow \mathrm{SL}$ which is alternative to [KKV04, Prop. 3.12]. This we do by utilizing Pontryagin duality for semilattices [HMS74]. We start by recalling the definition of a modal algebra.

Definition 2.1. A modal algebra is a pair $(B, \square)$ where $B$ is a boolean algebra and $\square$ is a unary function on $B$ preserving finite meets. A modal algebra homomorphism between modal algebras $\left(B_{1}, \square_{1}\right)$ and $\left(B_{2}, \square_{2}\right)$ is a boolean homomorphism $\alpha: B_{1} \rightarrow B_{2}$ such that $\alpha\left(\square_{1} a\right)=\square_{2} \alpha(a)$ for each $a \in B_{1}$. Let MA be the category of modal algebras and modal algebra homomorphisms.

A subset of a topological space $X$ is clopen if it is both closed and open, and $X$ is zero-dimensional if $X$ has a basis of clopen sets. A Stone space is a zero-dimensional compact Hausdorff space.

For a binary relation $R$ on $X$, we write

$$
R[x]:=\{y \in X \mid x R y\} \text { and } R^{-1}[U]:=\{x \in X \mid \exists u \in U \text { with } x R u\}
$$

for the $R$-image of $x \in X$ and $R$-inverse image of $U \subseteq X$.

Definition 2.2. A descriptive frame is a pair $(X, R)$ where $X$ is a Stone space and $R$ is a binary relation on $X$ such that $R[x]$ is closed for each $x \in X$ and $R^{-1}[U]$ is clopen for each clopen $U \subseteq X$.

Such relations are often called continuous relations for the following reason. Let $\mathcal{V}(X)$ be the Vietoris space of $X$. We recall (see, e.g., [Joh82, Sec. III.4]) that $\mathcal{V}(X)$ is the set of closed subsets of $X$ topologized by the subbasis $\left\{\square_{U}, \nabla_{V} \mid U, V\right.$ open in $\left.X\right\}$ where

$$
\square_{U}=\{F \in \mathcal{V}(X) \mid F \subseteq U\} \text { and } \diamond_{V}=\{F \in \mathcal{V}(X) \mid F \cap V \neq \varnothing\} .
$$

Then $R$ is continuous iff the associated map $\rho_{R}: X \rightarrow \mathcal{V}(X)$, given by $\rho_{R}(x)=R[x]$, is a well-defined continuous map (that $\rho_{R}$ is well defined follows from (i), and that it is continuous from (ii)).

Let DFr be the category of descriptive frames and continuous p-morphisms, where a p-morphism between $\left(X_{1}, R_{1}\right)$ and $\left(X_{2}, R_{2}\right)$ is a map $f: X_{1} \rightarrow X_{2}$ satisfying $f\left[R_{1}[x]\right]=$ $R_{2}[f(x)]$ for each $x \in X_{1}$.

Theorem 2.3 (Jónsson-Tarski duality). MA is dually equivalent to DFr.

Jónsson-Tarski duality generalizes Stone duality between the category BA of boolean algebras and boolean homomorphisms and the category Stone of Stone spaces and continuous maps. We recall that the contravariant functors $\mathfrak{u f}: \mathrm{BA} \rightarrow{ }^{\mathrm{op}}$ Stone and $\mathfrak{c l o p}:$ Stone $\rightarrow^{\mathrm{op}}$ BA yielding Stone duality are constructed as follows. ${ }^{3}$ The functor $\mathfrak{u} \mathfrak{f}$ assigns to each boolean algebra $A$ the set $\mathfrak{u f}(A)$ of ultrafilters of $A$ topologized by the basis $\left\{\beta_{A}(a) \mid a \in A\right\}$ where

$$
\beta_{A}(a)=\{x \in \mathfrak{u f}(A) \mid a \in x\} .
$$

To each boolean homomorphism $\alpha: A \rightarrow B$, the functor $\mathfrak{u f}$ assigns $\mathfrak{u f}(\alpha):=\alpha^{-1}: \mathfrak{u f}(B) \rightarrow$ $\mathfrak{u f}(A)$. The functor clop assigns to each Stone space $X$ the boolean algebra $\mathfrak{c l o p}(X)$ of

\footnotetext{
${ }^{3}$ To easily distinguish between covariant and contravariant functors, following the suggestion of one of the referees, we write $F: C \rightarrow^{\text {op }} \mathrm{D}$ for a contravariant functor $F$.
} 
clopen subsets of $X$, and to each continuous map $f: X \rightarrow Y$ the boolean homomorphism $\mathfrak{c l o p}(f):=f^{-1}: \mathfrak{c l o p}(Y) \rightarrow \mathfrak{c l o p}(X)$.

One unit $\beta: 1_{\mathrm{BA}} \rightarrow \mathfrak{c l o p} \circ \mathfrak{u f}$ of this dual equivalence is given by the Stone maps $\beta_{A}: A \rightarrow \mathfrak{c l o p}(\mathfrak{u f}(A))$ for $A \in \mathrm{BA}$, and the other unit $\eta: 1_{\text {Stone }} \rightarrow \mathfrak{u f} \circ \mathfrak{c l o p}$ by the homeomorphisms $\eta_{X}: X \rightarrow \mathfrak{u f}(\mathfrak{c l o p}(X))$ for $X \in$ Stone, which are given by

$$
\eta_{X}(x)=\{U \in \mathfrak{c l o p}(X) \mid x \in U\} .
$$

These functors naturally generalize to yield Jónsson-Tarski duality. As we pointed out in the introduction, an alternative approach to Jónsson-Tarski duality is by lifting Stone duality using algebra/coalgebra methods. We recall that the Vietoris construction extends to an endofunctor $\mathcal{V}:$ Stone $\rightarrow$ Stone by sending a continuous map $f: X \rightarrow Y$ to $\mathcal{V}(f): \mathcal{V}(X) \rightarrow \mathcal{V}(Y)$ given by $\mathcal{V}(f)(G)=f[G]$ for each $G \in \mathcal{V}(X)$. We next consider the category Coalg $(\mathcal{V})$ of coalgebras for $\mathcal{V}$. For this we recall the notion of a coalgebra for an endofunctor (see, e.g., [Ven07, Def. 9.1]).

\section{Definition 2.4.}

(1) A coalgebra for an endofunctor $\mathcal{T}: \mathrm{C} \rightarrow \mathrm{C}$ is a pair $(A, f)$ where $A$ is an object of the category $\mathrm{C}$ and $f: A \rightarrow \mathcal{T}(A)$ is a C-morphism.

(2) A morphism between two coalgebras $\left(A_{1}, f_{1}\right)$ and $\left(A_{2}, f_{2}\right)$ for $\mathcal{T}$ is a C-morphism $\alpha: A_{1} \rightarrow A_{2}$ such that the following square is commutative.

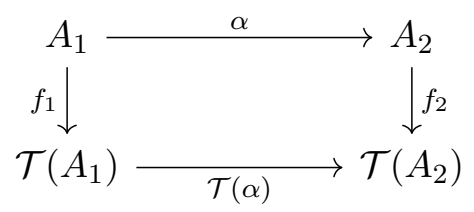

(3) Let Coalg $(\mathcal{T})$ be the category whose objects are coalgebras for $\mathcal{T}$ and whose morphisms are morphisms of coalgebras.

The dual endofunctor $\mathcal{K}: \mathrm{BA} \rightarrow \mathrm{BA}$ of the Vietoris endofunctor $\mathcal{V}:$ Stone $\rightarrow$ Stone was described in [KKV04]. Let SL be the category of meet-semilattices with top and meethomomorphisms preserving top. Then $\mathcal{K}$ is the composition $\mathcal{L U}$, where $\mathcal{L}: S L \rightarrow$ BA is the left adjoint of the forgetful functor $\mathcal{U}: \mathrm{BA} \rightarrow \mathrm{SL}$. In [KKV04, Prop. 3.12] the left adjoint is constructed algebraically, by taking the free boolean algebra $F$ over the underlying set of $M \in \mathrm{SL}$ and then taking the quotient of $F$ by the relations remembering that $M$ is a meet-semilattice with top. We give an alternative description of $\mathcal{L}$, which utilizes Pontryagin duality for semilattices [HMS74], which we briefly recall next.

Let StoneSL be the category whose objects are topological meet-semilattices, where the topology is a Stone topology, and whose morphisms are continuous meet-homomorphisms. Pontryagin duality for SL establishes a dual equivalence between SL and StoneSL. The contravariant functor $(-)^{*}: \mathrm{SL} \rightarrow{ }^{\mathrm{op}}$ StoneSL sends $M$ to its dual $M^{*}:=\operatorname{hom}_{\mathrm{SL}}(M, 2)$, where $2=\{0,1\}$ is the two-element chain and meet on $M^{*}$ is pointwise meet. If 2 is given the discrete topology and $2^{M}$ the product topology, then $M^{*}$ is easily seen to be a closed subspace of $2^{M}$, and so the subspace topology is a Stone topology. Moreover, pointwise meet is continuous, and hence $M^{*} \in$ StoneSL. On morphisms, if $\sigma: M \rightarrow N$ is an SL-morphism, then $\sigma^{*}: N^{*} \rightarrow M^{*}$ is defined by $\sigma^{*}(\gamma)=\gamma \circ \sigma$. The contravariant functor in the other direction sends $A \in$ StoneSL to $A^{*}:=\operatorname{hom}_{\text {StoneSL }}(A, 2)$ and $\sigma: A \rightarrow B$ to $\sigma^{*}: B^{*} \rightarrow A^{*}$, defined in the same way as the previous functor. Finally, one natural isomorphism sends each $M \in \mathrm{SL}$ to its double dual $M^{* *}$ by sending $m$ to the map $\sigma \mapsto \sigma(m)$ for each $m \in M$ 
and $\sigma \in M^{*}$. The other natural isomorphism sends each $A \in$ StoneSL to its double dual $A^{* *}$ and is given by the same formula.

Theorem 2.5. Associating with each $M \in \mathrm{SL}$ the boolean algebra $\operatorname{clop}\left(M^{*}\right)$ of clopen subsets of its dual $M^{*}$ yields an alternative description of the functor $\mathcal{L}: \mathrm{SL} \rightarrow \mathrm{BA}$ that is left adjoint to the forgetful functor $\mathcal{U}: \mathrm{BA} \rightarrow \mathrm{SL}$.

Proof. Let $M \in \mathrm{SL}$. Define $i_{M}: M \rightarrow \operatorname{clop}\left(M^{*}\right)$ by $i_{M}(m)=\left\{\sigma \in M^{*} \mid \sigma(m)=1\right\}$. It is straightforward to see that $i_{M}$ is a well-defined SL-morphism. By [Mac71, p. 89] it is enough to show that for each $A \in \mathrm{BA}$ and SL-morphism $\gamma: M \rightarrow A$ there is a unique BA-morphism $\tau: \mathfrak{c l o p}\left(M^{*}\right) \rightarrow A$ such that $\tau \circ i_{M}=\gamma$.

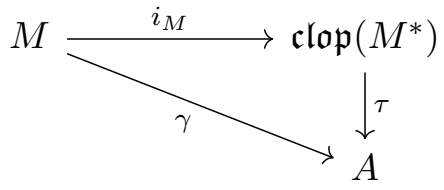

The map $\gamma^{*}: \operatorname{hom}_{\mathrm{SL}}(A, 2) \rightarrow M^{*}$ is continuous, so its restriction $\gamma^{*}: \operatorname{hom}_{\mathrm{BA}}(A, 2) \rightarrow M^{*}$ is continuous. Therefore, $\mathfrak{c l o p}\left(\gamma^{*}\right): \mathfrak{c l o p}\left(M^{*}\right) \rightarrow \mathfrak{c l o p}\left(\operatorname{hom}_{\mathrm{BA}}(A, 2)\right)$ is a BA-morphism. If $m \in M$, then

$$
\begin{aligned}
\mathfrak{c l o p}\left(\gamma^{*}\right)\left(i_{M}(m)\right) & =\left(\gamma^{*}\right)^{-1}\left(i_{M}(m)\right)=\left\{\sigma \in M^{*} \mid \gamma^{*}(\sigma) \in i_{M}(m)\right\} \\
& =\left\{\sigma \in M^{*} \mid \sigma(\gamma(m))=1\right\} .
\end{aligned}
$$

Let $\tau: \mathfrak{c l o p}\left(M^{*}\right) \rightarrow A$ be the composition of $\mathfrak{c l o p}\left(\gamma^{*}\right)$ with the inverse of the natural isomorphism $\beta_{A}: A \rightarrow \mathfrak{c l o p}\left(\operatorname{hom}_{\mathrm{BA}}(A, 2)\right)$ of Stone duality which sends $a \in A$ to $\left\{\sigma \in \operatorname{hom}_{\mathrm{BA}}(A, 2) \mid \sigma(a)=1\right\} .^{4}$ Then

$$
\tau\left(i_{M}(m)\right)=\beta_{A}^{-1} \mathfrak{c l o p}\left(\gamma^{*}\right)\left(i_{M}(m)\right)=\beta_{A}^{-1}\left(\left\{\sigma \in M^{*} \mid \sigma(\gamma(m))=1\right\}\right)=\gamma(m),
$$

so $\tau \circ i_{M}=\gamma$. Finally, uniqueness of $\tau$ follows since $i_{M}[M]$ generates $\mathfrak{c l o p}\left(M^{*}\right)$ as a boolean algebra.

Remark 2.6. To see how $\mathcal{L}$ acts on morphisms, if $\sigma: M \rightarrow N$ is an SL-morphism, then $\sigma^{*}: N^{*} \rightarrow M^{*}$ is a continuous SL-morphism between Stone spaces, so $\mathfrak{c l o p}\left(\sigma^{*}\right): \mathfrak{c l o p}\left(M^{*}\right) \rightarrow$ $\mathfrak{c l o p}\left(N^{*}\right)$ is a BA-morphism by Stone duality. We then set $\mathcal{L}(\sigma)=\mathfrak{c l o p}\left(\sigma^{*}\right): \mathcal{L}(M) \rightarrow \mathcal{L}(N)$.

Let $\mathcal{K}=\mathcal{L U}$. Then $\mathcal{K}$ is an endofunctor on BA.

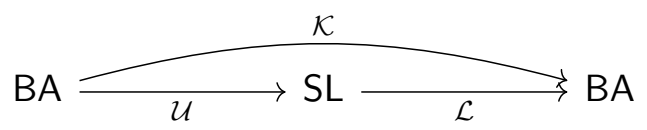

Let $\operatorname{Alg}(\mathcal{K})$ be the category of algebras for $\mathcal{K}$ (see [AHS06, Def. 5.37]). We recall that for an endofunctor $\mathcal{T}$, algebras for $\mathcal{T}$ are defined by reversing the arrows in the definition of coalgebras for $\mathcal{T}$. Since $\mathcal{K}$ is dual to $\mathcal{V}$, we have that $\operatorname{Alg}(\mathcal{K})$ is dually equivalent to Coalg $(\mathcal{V})$. Because $\operatorname{Alg}(\mathcal{K})$ is isomorphic to MA and Coalg $(\mathcal{V})$ is isomorphic to DFr, this gives an alternate proof of Jónsson-Tarski duality (see [KKV04]).

\footnotetext{
${ }^{4}$ Here we make the well-known identification of $\mathfrak{u f}(A)$ with $\operatorname{hom}_{\mathrm{BA}}(A, 2)$.
} 


\section{Two COnstructions of THE LeFt ADJoint $\mathcal{L}: \mathrm{CSL} \rightarrow$ CABA}

Let KFr be the category of Kripke frames and p-morphisms. Forgetting the topology of a descriptive frame yields the forgetful functor $\mathcal{U}: \mathrm{DFr} \rightarrow \mathrm{KFr}$. To describe the modal algebras corresponding to Kripke frames, we recall the notion of a completely multiplicative modal operator.

Definition 3.1. A modal operator $\square$ on a complete boolean algebra $B$ is completely multiplicative if $\square(\bigwedge S)=\bigwedge\{\square s \mid s \in S\}$ for each $S \subseteq B$. Let CAMA be the category whose objects are complete atomic modal algebras with completely multiplicative $\square$, and whose morphisms are complete modal algebra homomorphisms.

Theorem 3.2 (Thomason duality). CAMA is dually equivalent to KFr.

Thomason duality generalizes Tarski duality between CABA and Set the same way Jónsson-Tarski duality generalizes Stone duality. We recall that CABA is the category of complete atomic boolean algebras and complete boolean homomorphisms and Set is the category of sets and functions. The contravariant functors of Tarski duality are $\wp:$ Set $\rightarrow$ op CABA and $\mathfrak{a t}:$ CABA $\rightarrow^{\text {op }}$ Set. The functor $\wp$ assigns to each set $X$ the powerset $\wp(X)$ and to each function $f: X \rightarrow Y$ its inverse image $f^{-1}: \wp(Y) \rightarrow \wp(X)$. The functor at assigns to each $A \in \mathrm{CABA}$ its set of atoms. If $\alpha: A \rightarrow B$ is a complete boolean homomorphism, it has a left adjoint $\alpha^{*}: B \rightarrow A$, which sends atoms to atoms, and the functor at assigns to $\alpha$ the function $\alpha^{*}: \mathfrak{a t}(B) \rightarrow \mathfrak{a t}(A)$. One unit $\varepsilon: 1_{\text {Set }} \rightarrow \mathfrak{a t} \circ \wp$ of this dual equivalence is given by $\varepsilon_{X}(x)=\{x\}$ for each $x \in X \in$ Set, and the other unit $\vartheta: 1_{\text {CABA }} \rightarrow \wp \circ$ at by $\vartheta_{A}(a)=\downarrow a \cap \mathfrak{a t}(A)$ for each $a \in A \in \mathrm{CABA}$.

To derive Thomason duality from Tarski duality the same way Jónsson-Tarski duality was derived from Stone duality, we need to replace the Vietoris endofunctor $\mathcal{V}$ on Stone with the powerset endofunctor $\mathcal{P}$ on Set. We recall that the endofunctor $\mathcal{P}:$ Set $\rightarrow$ Set associates to each set $X$ its powerset $\mathcal{P}(X)$ and to each function $f: X \rightarrow Y$ the function $\mathcal{P}(f): \mathcal{P}(X) \rightarrow \mathcal{P}(Y)$ that maps each subset $S \subseteq X$ to its direct image $f[S]$. We also need to replace the endofunctor $\mathcal{K}: \mathrm{BA} \rightarrow \mathrm{BA}$ with an appropriate endofunctor $\mathcal{H}:$ CABA $\rightarrow$ CABA.

To describe $\mathcal{H}$, we need to construct the left adjoint to $\mathcal{U}:$ CABA $\rightarrow$ CSL, where CSL is the category of complete meet-semilattices and complete meet-homomorphisms. As in the previous section, this can be done purely algebraically or using duality. To construct $\mathcal{H}$ algebraically, we need that free objects exist in CABA. Care is needed here since it is a well-known result of Gaifman [Gai64] and Hales [Hal64] that free objects do not exist in the category of complete boolean algebras and complete boolean homomorphisms. On the other hand, free objects do exist in CABA, and this can be seen by observing that the Eilenberg-Moore algebras of the double contravariant powerset monad are exactly the objects of CABA [Tay02], and that categories of algebras for monads have free objects [AHS06, Prop. 20.7(2)].

A more concrete construction of free objects in CABA can be given utilizing the theory of canonical extensions. It is well known that free objects over any set exist in the category of complete and completely distributive lattices (see Markowski [Mar79] and Dwinger [Dwi81, Thm. 4.2]). By [BHJ21, Cor. 2.3], the free complete and completely distributive lattice over a set $X$ is the canonical extension of the free bounded distributive lattice over $X$. We show that the same is true in CABA. For this we need to recall the definition of a canonical extension of a boolean algebra. 
Definition 3.3. [JT51, GH01] A canonical extension of a boolean algebra $A$ is a complete boolean algebra $A^{\sigma}$ together with a boolean embedding $e: A \rightarrow A^{\sigma}$ satisfying:

(1) (Density) Each $x \in A^{\sigma}$ is a join of meets (and hence also a meet of joins) of $e[A]$.

(2) (Compactness) For $S, T \subseteq A$, from $\bigwedge e[S] \leq \bigvee e[T]$ it follows that $\bigwedge S_{0} \leq \bigvee T_{0}$ for some finite $S_{0} \subseteq S$ and $T_{0} \subseteq T$.

It is well known that canonical extensions are unique up to isomorphism, and that the correspondence $A \mapsto A^{\sigma}$ extends to a covariant functor $(-)^{\sigma}: \mathrm{BA} \rightarrow \mathrm{CABA}$. It can conveniently be described as the composition $\wp \circ \mathcal{U} \circ \mathfrak{u f}$, where $\mathcal{U}$ : Stone $\rightarrow$ Set is the forgetful functor.

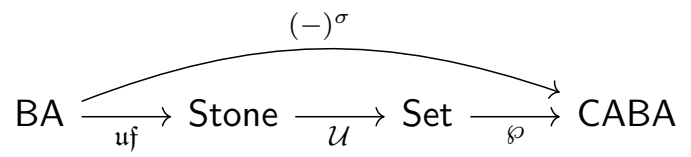

Thus, we can think of $A^{\sigma}$ as $\wp(\mathfrak{u f}(A))$ and of $e: A \rightarrow A^{\sigma}$ as the Stone map $\beta_{A}: A \rightarrow \wp(\mathfrak{u} \mathfrak{f}(A))$.

Theorem 3.4. Let $X$ be a set. The canonical extension of the free boolean algebra over $X$ is the free object in CABA over $X$.

Proof. Let $F$ be the free boolean algebra over $X, f: X \rightarrow F$ the associated map, and $e: F \rightarrow F^{\sigma}$ the boolean embedding into the canonical extension. We show that $\left(F^{\sigma}, e \circ f\right)$ has the universal mapping property in CABA. Let $A \in$ CABA and $g: X \rightarrow A$ be a function. Since $A$ is a boolean algebra, there is a unique boolean homomorphism $\varphi: F \rightarrow A$ with $\varphi \circ f=g$. This induces a map $\mathfrak{u f}(\varphi): \mathfrak{u f}(A) \rightarrow \mathfrak{u f}(F)$ given by $\mathfrak{u} \mathfrak{f}(\varphi)(y)=\varphi^{-1}(y)$. Define $\varphi_{+}: \mathfrak{a t}(A) \rightarrow \mathfrak{u f}(F)$ by $\varphi_{+}(x)=\varphi^{-1}(\uparrow x)$. If we identify atoms with the principal ultrafilters, we can think of $\varphi_{+}$as the restriction of $\mathfrak{u f}(\varphi)$ to $\mathfrak{a t}(A)$.

We identify $F^{\sigma}$ with $\wp(\mathfrak{u f}(F))$. Then $e: F \rightarrow F^{\sigma}$ becomes the Stone map $\beta_{F}$. The map $\varphi_{+}: \mathfrak{a t}(A) \rightarrow \mathfrak{u f}(F)$ yields a CABA-morphism $\wp\left(\varphi_{+}\right): F^{\sigma} \rightarrow \wp(\mathfrak{a t}(A))$. Since $A \in \mathrm{CABA}$, the map $\vartheta_{A}: A \rightarrow \wp(\mathfrak{a t}(A))$ is an isomorphism. We set $\psi=\vartheta_{A}^{-1} \circ \wp\left(\varphi_{+}\right)$. Clearly $\psi: F^{\sigma} \rightarrow A$ is a CABA-morphism. We show that $\vartheta_{A} \circ \varphi=\wp\left(\varphi_{+}\right) \circ e$.

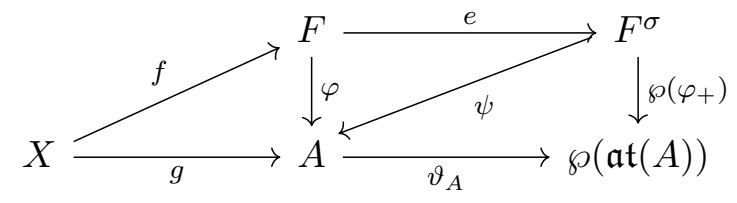

Let $a \in F$. Since $\vartheta_{A} \varphi(a)=\{x \in \mathfrak{a t}(A) \mid x \leq \varphi(a)\}$ and $e(a)=\beta_{F}(a)=\{y \in \mathfrak{u f}(F) \mid a \in y\}$, we have

$$
\begin{aligned}
\left(\wp\left(\varphi_{+}\right) \circ e\right)(a) & =\varphi_{+}^{-1} e(a)=\left\{x \in \mathfrak{a t}(A) \mid \varphi_{+}(x) \in e(a)\right\} \\
& =\left\{x \in \mathfrak{a t}(A) \mid a \in \varphi_{+}(x)\right\}=\left\{x \in \mathfrak{a t}(A) \mid a \in \varphi^{-1}(\uparrow x)\right\} \\
& =\{x \in \mathfrak{a t}(A) \mid x \leq \varphi(a)\}=\vartheta_{A} \varphi(a) .
\end{aligned}
$$

This shows that $\vartheta_{A} \circ \varphi=\wp\left(\varphi_{+}\right) \circ e$, so

$$
\psi \circ(e \circ f)=\vartheta_{A}^{-1} \circ \wp\left(\varphi_{+}\right) \circ e \circ f=\vartheta_{A}^{-1} \circ \vartheta_{A} \circ \varphi \circ f=\varphi \circ f=g .
$$

It is left to show uniqueness. Suppose that $\mu: F^{\sigma} \rightarrow A$ is a CABA-morphism satisfying $\mu \circ(e \circ f)=g$. Then $(\mu \circ e) \circ f=(\psi \circ e) \circ f=\varphi \circ f$. By uniqueness of $\varphi$, we have $\mu \circ e=\varphi=\psi \circ e$. Therefore, $\mu$ and $\psi$ agree on $e[F]$. Since $e[F]$ is dense in $F^{\sigma}$ and $\mu, \psi$ are CABA-morphisms, we conclude that $\mu=\psi$. 
We next show that the forgetful functor $\mathcal{U}: \mathrm{CABA} \rightarrow \mathrm{CSL}$ has a left adjoint $\mathcal{L}: \mathrm{CSL} \rightarrow$ CABA. Let $A \in \mathrm{CABA}$. We recall that a boolean congruence $\equiv$ on $A$ is a complete congruence if $a_{i} \equiv b_{i}$ for each $i \in I$ imply $\bigwedge\left\{a_{i} \mid i \in I\right\} \equiv \bigwedge\left\{b_{i} \mid i \in I\right\}$. It is well known that the quotient algebra $A / \equiv$ is also an object in CABA. As usual, for $a \in A$ we write $[a]$ for the equivalence class of $a$. Then the quotient map $\pi: A \rightarrow A / \equiv$, given by $a \mapsto[a]$, is a CABA-morphism.

Remark 3.5. There is a well-known one-to-one correspondence between congruences and ideals of a boolean algebra $A$, which associates to each boolean congruence $\equiv$ on $A$ the equivalence class of 0 . If $A \in \mathrm{CABA}$, this correspondence restricts to a one-to-one correspondence between complete congruences and principal ideals. In this case, the equivalence class of 0 is generated by the element $x=\bigvee\{a \Delta b \mid a \equiv b\}$, where $\Delta$ denotes symmetric difference in $A$.

If $M \in \mathrm{CSL}$, let $F(M)$ be the free object in CABA over the underlying set of $M$, and let $f_{M}: M \rightarrow F(M)$ be the associated map. We let $\equiv$ be the complete congruence on $F(M)$ generated by the relations:

$$
f_{M}(\bigwedge S) \equiv \bigwedge\left\{f_{M}(s) \mid s \in S\right\}, \text { where } S \subseteq M .
$$

We then set $\mathcal{L}(M)$ to be the quotient algebra $F(M) / \equiv$. Since $F(M) \in \mathrm{CABA}$ and $\equiv$ is a complete congruence, $\mathcal{L}(M) \in$ CABA. For $a \in M$, let $\square_{a}=\left[f_{M}(a)\right] \in \mathcal{L}(M)$. Let $\alpha_{M}: M \rightarrow \mathcal{L}(M)$ be the composition of the quotient map $\pi: F(M) \rightarrow \mathcal{L}(M)$ and $f_{M}$. Then $\alpha_{M}(a)=\square_{a}$ for each $a \in M$.

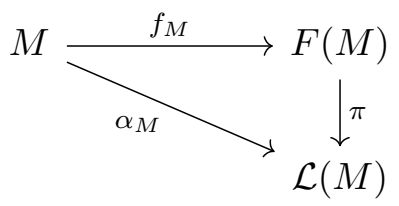

By the definition of $\equiv$ we see that $\square_{\bigwedge S}=\bigwedge\left\{\square_{s} \mid s \in S\right\}$ in $\mathcal{L}(M)$ for each $S \subseteq M$. Thus, $\alpha_{M}$ is a CSL-morphism.

Remark 3.6. In view of Remark 3.5, the equivalence class $[0] \in \mathcal{L}(M)$ is the principal ideal generated by the element

$$
\bigvee\left\{f_{M}(\bigwedge S) \triangle \bigwedge\left\{f_{M}(s) \mid s \in S\right\} \mid S \subseteq M\right\}
$$

Theorem 3.7. The correspondence $M \mapsto \mathcal{L}(M)$ defines a functor $\mathcal{L}: \mathrm{CSL} \rightarrow \mathrm{CABA}$ that is left adjoint to the forgetful functor $\mathcal{U}: \mathrm{CABA} \rightarrow \mathrm{CSL}$.

Proof. By [Mac71, p. 89] it is enough to show that for each $M \in$ CSL, $A \in$ CABA, and CSL-morphism $\gamma: M \rightarrow A$ there is a unique CABA-morphism $\tau: \mathcal{L}(M) \rightarrow A$ such that $\tau \circ \alpha_{M}=\gamma$. There is a unique CABA-morphism $\varphi: F(M) \rightarrow A$ with $\varphi \circ f_{M}=\gamma$. To see that $\varphi$ factors through $\equiv$, let $S \subseteq M$. Since $\gamma$ is a CSL-morphism, $\gamma(\bigwedge S)=\bigwedge\{\gamma(s) \mid s \in S\}$. Therefore,

$$
\varphi f_{M}(\bigwedge S)=\gamma(\bigwedge S)=\bigwedge \gamma[S]
$$

and

$$
\varphi\left(\bigwedge\left\{f_{M}(s) \mid s \in S\right\}\right)=\bigwedge\left\{\varphi f_{M}(s) \mid s \in S\right\}=\bigwedge\{\gamma(s) \mid s \in S\}=\bigwedge \gamma[S]
$$




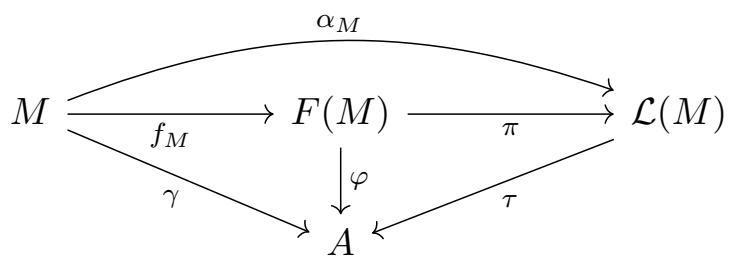

Thus, $\varphi f_{M}(\bigwedge S)=\varphi\left(\bigwedge\left\{f_{M}(s) \mid s \in S\right\}\right)$. This implies that $\equiv$ is contained in $\operatorname{ker}(\varphi)$, and hence $\varphi$ induces a CABA-morphism $\tau: \mathcal{L}(M) \rightarrow A$ with $\tau \circ \alpha_{M}=\gamma$. Since $\mathcal{L}(M)$ is generated by $\alpha_{M}[M]$ and $\tau$ is a CABA-morphism, $\tau$ is uniquely determined by the equation $\tau \circ \alpha_{M}=\gamma$.

Remark 3.8. To describe how $\mathcal{L}$ acts on morphisms, let $\gamma: M \rightarrow N$ be a CSL-morphism. Then $\alpha_{N} \circ \gamma: M \rightarrow \mathcal{L}(N)$ is a CSL-morphism, so there is a unique CABA-morphism $\mathcal{L}(\gamma): \mathcal{L}(M) \rightarrow \mathcal{L}(N)$ such that $\mathcal{L}(\gamma) \circ \alpha_{M}=\alpha_{N} \circ \gamma$.

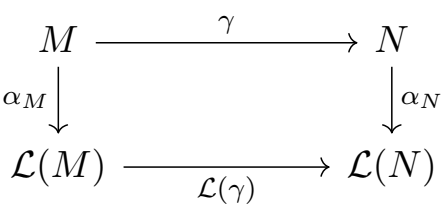

Therefore, if $a \in M$, then $\mathcal{L}(\gamma)\left(\square_{a}\right)=\mathcal{L}(\gamma) \alpha_{M}(a)=\alpha_{N} \gamma(a)=\square_{\gamma(a)}$.

We conclude this section by giving an alternative construction of the left adjoint of the forgetful functor CABA $\rightarrow$ CSL. In parallel to Theorem 2.5, we can replace SL by CSL and Stone duality by Tarski duality. Then for $M \in \mathrm{CSL}$ we can consider $\mathcal{L}(M)$ to be $\wp\left(\operatorname{hom}_{\mathrm{CSL}}(M, 2)\right)$. If $\sigma \in \operatorname{hom}_{\mathrm{CSL}}(M, 2)$, then $\sigma^{-1}(1)$ is a filter of $M$. Since $\sigma$ preserves arbitrary meets, letting $a=\bigwedge \sigma^{-1}(1)$ yields $\sigma^{-1}(1)=\uparrow a$. Conversely, if $a \in M$, then defining $\sigma_{a}$ by

$$
\sigma_{a}(m)= \begin{cases}1 & \text { if } a \leq m \\ 0 & \text { otherwise }\end{cases}
$$

yields $\sigma_{a} \in \operatorname{hom}_{\mathrm{CSL}}(M, 2)$. Because this correspondence reverses the order, there is an order-reversing bijection $f: M \rightarrow \operatorname{hom}_{\mathrm{CSL}}(M, 2)$ sending $a$ to $\sigma_{a}$. Thus, $\wp(f)$ : $\wp\left(\operatorname{hom}_{\mathrm{CSL}}(M, 2)\right) \rightarrow \wp(M)$ is a CABA-isomorphism. If $i_{M}: M \rightarrow \wp\left(\operatorname{hom}_{\mathrm{CSL}}(M, 2)\right)$ is given by $i_{M}(m)=\left\{\sigma \in \operatorname{hom}_{\mathrm{CSL}}(M, 2) \mid \sigma(m)=1\right\}$, then we have the function $\iota_{M}=\wp(f) \circ i_{M}: M \rightarrow \wp(M)$ given by

$$
\begin{aligned}
\iota_{M}(m) & =\wp(f)\left(i_{M}(m)\right)=\left\{a \in M \mid f(a) \in i_{M}(m)\right\}=\{a \in M \mid f(a)(m)=1\} \\
& =\{a \in M \mid a \leq m\}=\downarrow m .
\end{aligned}
$$

Thus, we may set $\mathcal{L}(M)=\wp(M)$ and define $\iota_{M}: M \rightarrow \mathcal{L}(M)$ by $\iota(m)=\downarrow m$.

Theorem 3.9. Associating with each $M \in \mathrm{CSL}$ its powerset yields an alternative description of the functor $\mathcal{L}: \mathrm{CSL} \rightarrow \mathrm{CABA}$ that is left adjoint to the forgetful functor $\mathcal{U}: \mathrm{CABA} \rightarrow \mathrm{CSL}$.

Proof. Let $M \in \mathrm{CSL}$. Clearly the powerset of $M$ is an object in CABA. Define $\iota_{M}: M \rightarrow$ $\mathcal{L}(M)$ by $\iota_{M}(a)=\downarrow a$ for each $a \in M$. For each $S \subseteq M$, we have

$$
\bigwedge\left\{\iota_{M}(s) \mid s \in S\right\}=\bigcap\{\downarrow s \mid s \in S\}=\downarrow(\bigwedge S)=\iota_{M}(\bigwedge S) .
$$

Therefore, $\iota_{M}$ is a CSL-morphism. It is enough to show that for each $A \in \mathrm{CABA}$ and CSL-morphism $\gamma: M \rightarrow A$ there is a unique CABA-morphism $\tau: \mathcal{L}(M) \rightarrow A$ such that 
$\tau \circ \iota_{M}=\gamma$.

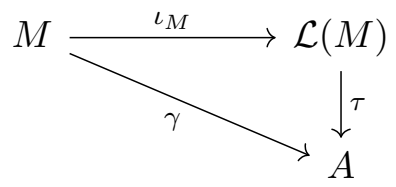

Let $\gamma^{*}$ be the left adjoint of $\gamma$, and consider its restriction $\gamma^{*}: \mathfrak{a t}(A) \rightarrow M$. Also recall that $\vartheta_{A}: A \rightarrow \wp(\mathfrak{a t}(A))$ is a CABA-isomorphism, hence so is $\vartheta_{A}^{-1}: \wp(\mathfrak{a t}(A)) \rightarrow A$ which is given by $\vartheta_{A}^{-1}(S)=\bigvee S$ for $S \subseteq \mathfrak{a t}(A)$. We set $\tau=\vartheta_{A}^{-1} \circ \wp\left(\gamma^{*}\right): \mathcal{L}(M) \rightarrow A$.

$$
\mathcal{L}(M)=\wp(M) \underset{\wp\left(\gamma^{*}\right)}{\wp} \wp(\mathfrak{a t}(A)) \underset{\vartheta_{A}^{-1}}{\longrightarrow} A
$$

Then $\tau$ is the composition of two CABA-morphisms, so is a CABA-morphism. Moreover, for $S \subseteq M$, we have

$$
\tau(S)=\vartheta_{A}^{-1} \wp\left(\gamma^{*}\right)(S)=\vartheta_{A}^{-1}\left(\left\{x \in \mathfrak{a t}(A) \mid \gamma^{*}(x) \in S\right\}\right)=\bigvee\left\{x \in \mathfrak{a t}(A) \mid \gamma^{*}(x) \in S\right\} .
$$

Thus, for $a \in M$, we have

$$
\tau\left(\iota_{M}(a)\right)=\tau(\downarrow a)=\bigvee\left\{x \in \mathfrak{a t}(A) \mid \gamma^{*}(x) \leq a\right\}=\bigvee\{x \in \mathfrak{a t}(A) \mid x \leq \gamma(a)\}=\gamma(a)
$$

since $A$ is atomic. To show that $\tau$ is uniquely determined by the equation $\tau \circ \iota_{M}=\gamma$, it is enough to show that $\mathcal{L}(M)$ is generated as a complete boolean algebra by $\iota_{M}[M]$. Since each $S \subseteq M$ is the union of singletons, this follows from the equation

$$
\{a\}=\downarrow a \backslash\{b \mid b<a\}=\downarrow a \backslash \bigcup\{\downarrow b \mid b<a\}=\iota_{M}(a) \wedge \neg \bigvee\left\{\iota_{M}(b) \mid b<a\right\}
$$

Remark 3.10. It is worth mentioning that the equation $\{a\}=\iota_{M}(a) \wedge \neg \bigvee\left\{\iota_{M}(b) \mid b<a\right\}$ above allows an alternate description of $\tau: \mathcal{L}(M) \rightarrow A$ that does not involve atoms. Indeed, since $\tau$ is a CABA-morphism, we have

$$
\begin{aligned}
\tau(\{a\}) & =\tau\left(\iota_{M}(a) \wedge \neg \bigvee\left\{\iota_{M}(b) \mid b<a\right\}\right)=\tau \iota_{M}(a) \wedge \neg \bigvee\left\{\tau \iota_{M}(b) \mid b<a\right\} \\
& =\gamma(a) \wedge \neg \bigvee\{\gamma(b) \mid b<a\} .
\end{aligned}
$$

Thus, for each $S \subseteq M$, we have

$$
\tau(S)=\bigvee\{\tau(\{a\}) \mid a \in S\}=\bigvee\{\gamma(a) \wedge \neg \bigvee\{\gamma(b) \mid b<a\} \mid a \in S\}
$$

Remark 3.11. To describe how $\mathcal{L}$ acts on morphisms, let $\gamma: M \rightarrow N$ be a CSL-morphism. Then $\iota_{N} \circ \gamma: M \rightarrow \mathcal{L}(N)$ is a CSL-morphism, so there is a unique CABA-morphism $\mathcal{L}(\gamma): \mathcal{L}(M) \rightarrow \mathcal{L}(N)$ such that $\mathcal{L}(\gamma) \circ \iota_{M}=\iota_{N} \circ \gamma$.

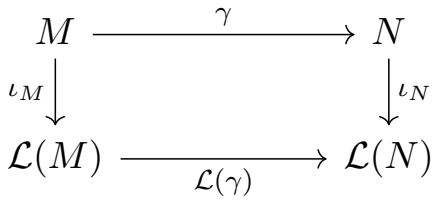

Therefore, if $a \in M$, then $\mathcal{L}(\gamma)(\downarrow a)=\mathcal{L}(\gamma) \iota_{M}(a)=\iota_{N} \gamma(a)=\downarrow \gamma(a)$. 


\section{Coalgebraic approach to Thomason duality}

Definition 4.1. Let $\mathcal{H}: \mathrm{CABA} \rightarrow \mathrm{CABA}$ be the composition $\mathcal{H}=\mathcal{L U}$.

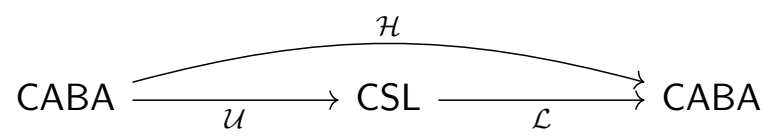

Remark 4.2. In Theorems 3.7 and 3.9 we have given two alternative constructions of $\mathcal{L}: \mathrm{CSL} \rightarrow$ CABA. Thus, we have two alternative descriptions of $\mathcal{H}: \mathrm{CABA} \rightarrow \mathrm{CABA}$. For $A \in \mathrm{CABA}$ we can think of $\mathcal{H}(A)$ as the powerset of $A$ (Theorem 3.9) or as the quotient of the free object in CABA over $A$ (Theorem 3.7). The resulting two functors are naturally isomorphic. In this section we will always assume that $\mathcal{H}(A)$ is the powerset of $A$, but will indicate how the corresponding result can be proved if we think of $\mathcal{H}(A)$ as the quotient of the free object in CABA over $A$.

We show that the diagram in Figure 1 is commutative up to natural isomorphism. The horizontal arrows in the diagram represent the contravariant functors at: Set $\rightarrow{ }^{\mathrm{op}}$ CABA and $\wp:$ Set $\rightarrow{ }^{\mathrm{op}}$ CABA of Tarski duality, whereas the vertical arrows represent the endofunctors $\mathcal{H}$ and $\mathcal{P}$ on CABA and Set, respectively. This together with standard algebra/coalgebra machinery then allows us to prove that $\operatorname{Alg}(\mathcal{H})$ is dually equivalent to $\operatorname{Coalg}(\mathcal{P})$, thus yielding an alternate proof of Thomason duality.

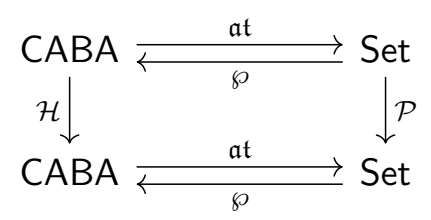

FiguRe 1

\section{Theorem 4.3.}

(1) $\mathcal{H} \circ \wp=\wp \circ \mathcal{P}$.

(2) at $\circ \mathcal{H}$ is naturally isomorphic to $\mathcal{P} \circ$ at.

Proof. (1) If $X \in$ Set, then $\wp \mathcal{P}(X)$ and $\mathcal{H} \wp(X)$ are both objects in CABA obtained by taking the double powerset of $X$ ordered by inclusion. We show that the two compositions also agree on morphisms. Let $f: X \rightarrow Y$ be a map. It is sufficient to show that $\wp \mathcal{P}(f)(\downarrow S)=\mathcal{H} \wp(f)(\downarrow S)$ for each $S \subseteq Y$. By Remark 3.11, we have

$$
\mathcal{H} \wp(f)(\downarrow S)=\downarrow \wp(f)(S)=\downarrow f^{-1}(S) .
$$

On the other hand,

$$
\begin{aligned}
\wp \mathcal{P}(f)(\downarrow S) & =\mathcal{P}(f)^{-1}(\downarrow S)=\{T \in \wp(X) \mid \mathcal{P}(f)(T) \in \downarrow S\} \\
& =\{T \in \wp(X) \mid f[T] \subseteq S\}=\left\{T \in \wp(X) \mid T \subseteq f^{-1}(S)\right\}=\downarrow f^{-1}(S) .
\end{aligned}
$$

Thus, $\wp \mathcal{P}(f)(\downarrow S)=\mathcal{H} \wp(f)(\downarrow S)$, completing the proof.

(2) follows from (1) since the horizontal arrows in the diagram in Figure 1 form a dual equivalence.

Remark 4.4. If we use the alternative description of $\mathcal{H}$, then Theorem 4.3(1) should be phrased as $\mathcal{H} \circ \wp$ is naturally isomorphic to $\wp \circ \mathcal{P}$. The natural isomorphism $\xi: \mathcal{H} \circ \wp \rightarrow \wp \circ \mathcal{P}$ is given on the generators of $\mathcal{H} \wp(X)$ by $\xi_{X}\left(\square_{S}\right)=\downarrow S$ for each $S \subseteq X \in$ Set. 
In the next remark we give an explicit description of the natural isomorphism $\zeta: \mathfrak{a t} \circ \mathcal{H} \rightarrow$ $\mathcal{P} \circ$ at. This will be used in Remark 4.11.

Remark 4.5. For $A \in \mathrm{CABA}$ define $\zeta_{A}: \mathfrak{a t} \mathcal{H}(A) \rightarrow \mathcal{P a t}(A)$ by

$$
\zeta_{A}(\{a\})=\{x \in \mathfrak{a t}(A) \mid x \leq a\}
$$

for each $a \in A$. Since $\mathcal{H} \circ \wp=\wp \circ \mathcal{P}$ and $\varepsilon, \vartheta$ are natural isomorphisms of Tarski duality, we have that the composition $\mathfrak{a t} \mathcal{H}\left(\vartheta_{A}\right) \circ \varepsilon_{\mathcal{P a t}(A)}$ is a bijection.

$$
\mathcal{P a t}(A) \stackrel{\varepsilon_{\mathcal{P a t}(A)}}{\longrightarrow} \mathfrak{a t} \wp \mathcal{P} \mathfrak{a t}(A)=\mathfrak{a t} \mathcal{H} \wp \mathfrak{a t}(A) \stackrel{\mathfrak{a t} \mathcal{H}\left(\vartheta_{A}\right)}{\longrightarrow} \mathfrak{a t} \mathcal{H}(A)
$$

We show that for each $a \in A$ we have

$$
\left(\mathfrak{a t H}\left(\vartheta_{A}\right) \circ \varepsilon_{\mathcal{P a t}(A)}\right)(\{x \in \mathfrak{a t}(A) \mid x \leq a\})=\{a\} .
$$

Since $\varepsilon_{\mathcal{P a t}(A)}(\{x \in \mathfrak{a t}(A) \mid x \leq a\})=\{\{x \in \mathfrak{a t}(A) \mid x \leq a\}\}$, it is sufficient to prove that

$$
\mathfrak{a t} \mathcal{H}\left(\vartheta_{A}\right)(\{\{x \in \mathfrak{a t}(A) \mid x \leq a\}\})=\{a\} .
$$

It follows from Remark 3.10 that

$$
\begin{aligned}
\mathcal{H}\left(\vartheta_{A}\right)(\{a\}) & =\downarrow \vartheta_{A}(a) \wedge \neg \bigvee\left\{\downarrow \vartheta_{A}(b) \mid b<a\right\} \\
& =\downarrow\{x \in \mathfrak{a t}(A) \mid x \leq a\} \backslash \bigcup\{\downarrow\{x \in \mathfrak{a t}(A) \mid x \leq b\} \mid b<a\} \\
& =\{\{x \in \mathfrak{a t}(A) \mid x \leq a\}\} .
\end{aligned}
$$

In particular, $\{\{x \in \mathfrak{a t}(A) \mid x \leq a\}\} \leq \mathcal{H}\left(\vartheta_{A}\right)(\{a\})$, and so

$$
\mathfrak{a t} \mathcal{H}\left(\vartheta_{A}\right)(\{\{x \in \mathfrak{a t}(A) \mid x \leq a\}\}) \leq\{a\}
$$

because $\mathfrak{a t} \mathcal{H}\left(\vartheta_{A}\right)$ is left adjoint to $\mathcal{H}\left(\vartheta_{A}\right)$. Therefore, $\mathfrak{a t} \mathcal{H}\left(\vartheta_{A}\right)(\{\{x \in \mathfrak{a t}(A) \mid x \leq a\}\})=\{a\}$ since both sides of the last inequality are atoms. Thus, $\zeta_{A}=\left(\mathfrak{a t} \mathcal{H}\left(\vartheta_{A}\right) \circ \varepsilon_{\mathcal{P a t}(A)}\right)^{-1}$, and hence $\zeta$ is a natural isomorphism.

We next utilize Theorem 4.3 and standard algebra/coalgebra machinery to show that Tarski duality lifts to a dual equivalence between $\operatorname{Alg}(\mathcal{H})$ and $\operatorname{Coa} \lg (\mathcal{P})$. We start with the following well-known result (see, e.g., [Ven07, Sec. 9]). Since we will be using the functors establishing the isomorphism of Theorem 4.6 in Remark 4.13, we sketch the proof.

Theorem 4.6. KFr is isomorphic to $\operatorname{Coalg}(\mathcal{P})$.

Proof (Sketch). To each Kripke frame $\mathfrak{F}=(X, R)$ we associate the coalgebra $\rho_{R}: X \rightarrow \mathcal{P}(X)$ defined by $\rho_{R}(x)=R[x]$. If $f: X_{1} \rightarrow X_{2}$ is a p-morphism between Kripke frames $\left(X_{1}, R_{1}\right)$ and $\left(X_{2}, R_{2}\right)$, then $f$ is also a morphism between the coalgebras $\left(X_{1}, \rho_{R_{1}}\right)$ and $\left(X_{2}, \rho_{R_{2}}\right)$. This defines a covariant functor $\mathcal{C}: \operatorname{KFr} \rightarrow \operatorname{Coalg}(\mathcal{P})$. To each coalgebra $(X, \rho)$ for $\mathcal{P}$, we associate the Kripke frame $\left(X, R_{\rho}\right)$ where $x R_{\rho} y$ iff $y \in \rho(x)$. If $f$ is a morphism between two coalgebras $\left(X_{1}, \rho_{1}\right)$ and $\left(X_{2}, \rho_{2}\right)$, then $f$ is also a p-morphism between the Kripke frames $\left(X_{1}, R_{\rho_{1}}\right)$ and $\left(X_{2}, R_{\rho_{2}}\right)$. This defines a covariant functor $\mathcal{F}: \operatorname{Coalg}(\mathcal{P}) \rightarrow \mathrm{KFr}$. It is straightforward to see that $R=R_{\rho_{R}}$ for each $(X, R) \in \operatorname{KFr}$ and $\rho=\rho_{R_{\rho}}$ for each $(X, \rho) \in \operatorname{Coalg}(\mathcal{P})$. Thus, the functors $\mathcal{C}$ and $\mathcal{F}$ yield an isomorphism of $\operatorname{KFr}$ and $\operatorname{Coalg}(\mathcal{P})$.

We next show that CAMA is isomorphic to $\operatorname{Alg}(\mathcal{H})$. This is parallel to the well-known fact that MA is isomorphic to $\operatorname{Alg}(\mathcal{K})$ (see, e.g., [KKV04, Cor. 3.11]).

Theorem 4.7. CAMA is isomorphic to $\operatorname{Alg}(\mathcal{H})$. 
Proof. Let $(A, \square) \in$ CAMA. Since $\square: A \rightarrow A$ is a CSL-morphism, by Theorem 3.9, there is a unique CABA-morphism $\tau_{\square}: \mathcal{H}(A) \rightarrow A$ such that $\tau_{\square}(\downarrow a)=\square a$ for each $a \in A$. Therefore, $\left(A, \tau_{\square}\right) \in \operatorname{Alg}(\mathcal{H})$. Let $\alpha: A_{1} \rightarrow A_{2}$ be a CAMA-morphism and $a \in A_{1}$. Since $\alpha\left(\square_{1} a\right)=\square_{2} \alpha(a)$, by Remark 3.11,

$$
\tau_{\square_{2}} \mathcal{H}(\alpha)(\downarrow a)=\tau_{\square_{2}}(\downarrow \alpha(a))=\square_{2} \alpha(a)=\alpha\left(\square_{1} a\right)=\alpha \tau_{\square_{1}}(\downarrow a) .
$$

Because $\mathcal{H}(A)$ is generated by $\{\downarrow a \mid a \in A\}$, we obtain that $\tau_{\square_{2}} \circ \mathcal{H}(\alpha)=\alpha \circ \tau_{\square_{1}}$. Therefore, $\alpha$ is also a morphism in $\operatorname{Alg}(\mathcal{H})$. This defines a covariant functor $\mathcal{A}:$ CAMA $\rightarrow \operatorname{Alg}(\mathcal{H})$.

Conversely, let $(A, \tau) \in \operatorname{Alg}(\mathcal{H})$ so $A \in \mathrm{CABA}$ and $\tau: \mathcal{H}(A) \rightarrow A$ is a CABA-morphism. If we define $\square_{\tau}$ on $A$ by $\square_{\tau} a=\tau(\downarrow a)$, it is easy to see that $\square_{\tau}$ is completely multiplicative, so $\left(A, \square_{\tau}\right) \in$ CAMA. Let $\alpha: A_{1} \rightarrow A_{2}$ be a morphism in $\operatorname{Alg}(\mathcal{H})$ and $a \in A_{1}$. By Remark 3.11,

$$
\square_{\tau_{2}} \alpha(a)=\tau_{2}(\downarrow \alpha(a))=\tau_{2} \mathcal{H}(\alpha)(\downarrow a)=\alpha \tau_{1}(\downarrow a)=\alpha\left(\square_{\tau_{1}} a\right) .
$$

Therefore, $\alpha$ is also a CAMA-morphism. This defines a covariant functor $\mathcal{M}: \operatorname{Alg}(\mathcal{H}) \rightarrow$ CAMA.

Let $(A, \square) \in$ CAMA. For $a \in A$, we have $\square_{\tau_{\square}} a=\tau_{\square}(\downarrow a)=\square a$. Therefore, $\square_{\tau_{\square}}=\square$. Next, let $(A, \tau) \in \operatorname{Alg}(\mathcal{H})$. For $a \in A$, we have $\tau_{\square_{\tau}}(\downarrow a)=\square_{\tau} a=\tau(\downarrow a)$. Since $\mathcal{H}(A)$ is generated by $\{\downarrow a \mid a \in A\}$, we obtain that $\tau_{\square_{\tau}}=\tau$. Thus, the functors $\mathcal{A}$ and $\mathcal{M}$ yield an isomorphism of CAMA and $\operatorname{Alg}(\mathcal{H})$.

Remark 4.8. If we use the alternative description of $\mathcal{H}$, then the previous theorem can be proved using Theorem 3.7 and Remark 3.8. The advantage of using this description of $\mathcal{H}$ lies in the suggestive definitions $\tau_{\square}\left(\square_{a}\right)=\square a$ and $\square_{\tau} a=\tau\left(\square_{a}\right)$.

We are ready to lift Tarski duality to a dual equivalence between $\operatorname{Alg}(\mathcal{H})$ and $\operatorname{Coalg}(\mathcal{P})$. For this we utilize [Jac17, Thm. 2.5.9] which states that, under certain conditions, adjunctions lift to adjunctions between categories of algebras. For our purposes, we require the following reformulation of [Jac17, Thm. 2.5.9] for dual equivalences.

Lemma 4.9. Let $\mathbb{S}: \mathrm{C} \rightarrow \mathrm{C}, \mathbb{T}: \mathrm{D} \rightarrow \mathrm{D}$ be two endofunctors and $\mathbb{Q}: \mathrm{C} \rightarrow{ }^{\mathrm{op}} \mathrm{D}, \mathbb{R}: \mathrm{D} \rightarrow{ }^{\mathrm{op}} \mathrm{C}$ two contravariant functors forming a dual equivalence. Suppose that $\mathbb{S R}$ and $\mathbb{R T}$ are naturally isomorphic (and hence so are $\mathbb{T} \mathbb{Q}$ and $\mathbb{Q S}$ ). Then $\mathbb{Q}$ and $\mathbb{R}$ lift to contravariant functors $\widehat{\mathbb{Q}}: \operatorname{Alg}(\mathbb{S}) \rightarrow^{\mathrm{op}} \operatorname{Coalg}(\mathbb{T})$ and $\widehat{\mathbb{R}}: \operatorname{Coalg}(\mathbb{T}) \rightarrow^{\mathrm{op}} \mathrm{Alg}(\mathbb{S})$ which yield a dual equivalence between $\operatorname{Alg}(\mathbb{S})$ and Coalg $(\mathbb{T})$.

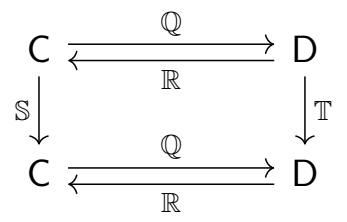

Theorem 4.3 and Lemma 4.9 then immediately give:

Theorem 4.10. Tarski duality between CABA and Set lifts to a dual equivalence between $\operatorname{Alg}(\mathcal{H})$ and $\operatorname{Coalg}(\mathcal{P})$.

Remark 4.11. By adapting the proof of [Jac17, Thm. 2.5.9], the contravariant functors $\widehat{\mathfrak{a t}}: \operatorname{Alg}(\mathcal{H}) \rightarrow^{\mathrm{op}} \operatorname{Coalg}(\mathcal{P})$ and $\widehat{\wp}: \operatorname{Coalg}(\mathcal{P}) \rightarrow^{\mathrm{op}} \operatorname{Alg}(\mathcal{H})$ lifting at $:$ CABA $\rightarrow^{\text {op }}$ Set and $\wp:$ Set $\rightarrow{ }^{\mathrm{op}}$ CABA can be defined as follows:

If $(A, f) \in \operatorname{Alg}(\mathcal{H})$, then $\left(\mathfrak{a t}(A), \zeta_{A} \circ \mathfrak{a t}(f)\right) \in \operatorname{Coalg}(\mathcal{P})$ where $\zeta_{A}$ is defined in Remark 4.5. Moreover, if $\alpha: A_{1} \rightarrow A_{2}$ is a morphism in $\operatorname{Alg}(\mathcal{H})$, then $\mathfrak{a t}(\alpha)$ is a morphism of the 
corresponding coalgebras. This defines the functor $\widehat{\mathfrak{a t}}: \operatorname{Alg}(\mathcal{H}) \rightarrow^{\mathrm{op}} \operatorname{Coalg}(\mathcal{P})$.

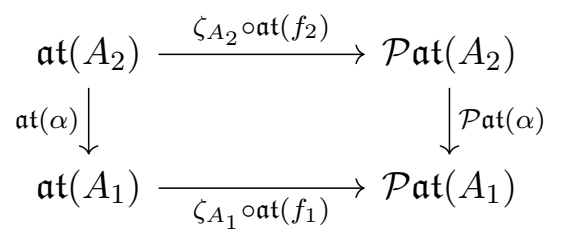

Let $(X, g) \in \operatorname{Coalg}(\mathcal{P})$. Then $\wp(g): \wp \mathcal{P}(X) \rightarrow \wp(X)$ is a CABA-morphism. Since $\mathcal{H} \circ \wp=\wp \circ \mathcal{P}$ (see Theorem 4.3(1)), we have that $(\wp(X), \wp(g)) \in \operatorname{Alg}(\mathcal{H})$. Moreover, if $h:\left(X_{1}, g_{1}\right) \rightarrow\left(X_{2}, g_{2}\right)$ is a Coalg $(\mathcal{P})$-morphism, then $\wp(h)$ is an $\operatorname{Alg}(\mathcal{H})$-morphism. This defines the functor $\widehat{\wp}: \operatorname{Coalg}(\mathcal{P}) \rightarrow{ }^{\text {op }} \operatorname{Alg}(\mathcal{H})$.

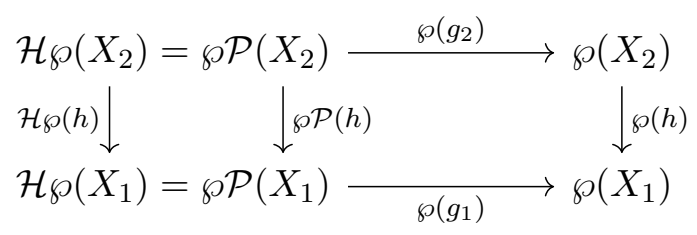

Remark 4.12. Putting Theorems 4.6, 4.7, and 4.10 together yields an alternate proof of Thomason duality. We recall that the contravariant functors establishing Thomason duality extend the contravariant functors of Tarski duality. Namely, the functor $\wp: \mathrm{KFr} \rightarrow{ }^{\mathrm{op}}$ CAMA associates to each $(X, R) \in \operatorname{KFr}$ the algebra $\left(\wp(X), \square_{R}\right) \in$ CAMA where $\square_{R}$ is defined by

$$
\square_{R}(S)=\{x \in X \mid R[x] \subseteq S\} .^{5}
$$

Also, $\wp$ associates to each KFr-morphism $f: X \rightarrow Y$ the CAMA-morphism $\wp(f): \wp(Y) \rightarrow$ $\wp(X)$ given by $\wp(f)=f^{-1}$. The functor at : CAMA $\rightarrow^{\mathrm{op}} \mathrm{KFr}$ associates to each $(A, \square) \in$ CAMA the Kripke frame $\left(\mathfrak{a t}(A), R_{\square}\right)$ where

$$
x R_{\square} y \text { iff } x \wedge \square \neg y=0 \text { iff }(\forall a \in A)(x \leq \square a \Rightarrow y \leq a) .
$$

Also, at associates to each CAMA-morphism $\alpha: A \rightarrow B$ the p-morphism at $(\alpha): \mathfrak{a t}(B) \rightarrow$ $\mathfrak{a t}(A)$ given by $\mathfrak{a t}(\alpha)=\alpha^{*}$.

We show that $\wp: \mathrm{KFr} \rightarrow{ }^{\mathrm{op}}$ CAMA is the composition

$$
\mathrm{KFr} \stackrel{\mathcal{C}}{\longrightarrow} \operatorname{Coalg}(\mathcal{P}) \stackrel{\widehat{\wp}}{\longrightarrow} \operatorname{Alg}(\mathcal{H}) \stackrel{\mathcal{M}}{\longrightarrow} \text { CAMA }
$$

and that at $:$ CAMA $\rightarrow{ }^{\mathrm{OP}} \mathrm{KFr}$ is the composition

$$
\mathrm{CAMA} \stackrel{\mathcal{A}}{\longrightarrow} \operatorname{Alg}(\mathcal{H}) \stackrel{\widehat{\mathfrak{a t}}}{\longrightarrow} \operatorname{Coalg}(\mathcal{P}) \stackrel{\mathcal{F}}{\longrightarrow} \mathrm{KFr} .
$$

Let $(X, R) \in \mathrm{KFr}$. Then

$$
\mathcal{M} \widehat{\wp} \mathcal{C}(X, R)=\mathcal{M} \widehat{\wp}\left(X, \rho_{R}\right)=\mathcal{M}\left(\wp(X), \wp\left(\rho_{R}\right)\right)=\left(\wp(X), \square_{\wp\left(\rho_{R}\right)}\right) .
$$

For $S \subseteq X$, we have

$$
\square_{\wp\left(\rho_{R}\right)} S=\wp\left(\rho_{R}\right)(\downarrow S)=\rho_{R}^{-1}(\downarrow S)=\left\{x \in X \mid \rho_{R}(x) \subseteq S\right\}=\{x \in X \mid R[x] \subseteq S\}=\square_{R} S .
$$

Thus, $\square_{\wp\left(\rho_{R}\right)}=\square_{R}$, so $\mathcal{M} \widehat{\wp} \mathcal{C}(X, R)=\left(\wp(X), \square_{R}\right)$, and hence $\mathcal{M} \widehat{\wp} \mathcal{C}=\wp$.

Let $(A, \square) \in$ CAMA . Then

$$
\mathcal{F} \widehat{\mathfrak{a t}} \mathcal{A}(A, \square)=\mathcal{F} \widehat{\mathfrak{a t}}\left(A, \tau_{\square}\right)=\mathcal{F}\left(\mathfrak{a t}(A), \zeta_{A} \circ \mathfrak{a t}\left(\tau_{\square}\right)\right)=\left(\mathfrak{a t}(A), R_{\zeta_{A} \circ \mathfrak{a t}\left(\tau_{\square}\right)}\right)
$$

\footnotetext{
${ }^{5}$ Thus, $\square_{R}$ is the predicate lifting for $\square$ composed with the coalgebra map $\rho_{R}: X \rightarrow \mathcal{P}(X)$.
} 
For $x, y \in \mathfrak{a t}(A)$, we have

$$
x R_{\zeta_{A} \circ \mathfrak{a t}\left(\tau_{\square}\right)} y \text { iff } y \in \zeta_{A} \mathfrak{a t}\left(\tau_{\square}\right)(x) \text { iff } y \in \zeta_{A}\left(\left(\tau_{\square}\right)^{*}(x)\right) .
$$

By Remark 3.10, for $a \in A$, we have $\tau_{\square}(\{a\})=\square a \wedge \neg \bigvee\{\square b \mid b<a\}$. Therefore, for each $x \in \mathfrak{a t}(A)$, we have

$$
x \leq \tau_{\square}(\{a\}) \text { iff } x \leq \square a \text { and } x \not \leq \square b \text { for each } b<a .
$$

Since $\square$ is completely multiplicative, $c:=\bigwedge\{a \in A \mid x \leq \square a\}$ is the least element satisfying $x \leq \square c$. Thus, $x \leq \tau_{\square}(\{c\})$. Consequently, $\left(\tau_{\square}\right)^{*}(x)=\{c\}$ since $\left(\tau_{\square}\right)^{*}$ is left adjoint to $\tau_{\square}$ and both $\left(\tau_{\square}\right)^{*}(x)$ and $\{c\}$ are atoms of $\mathcal{H}(A)$. It follows that

$$
y \in \zeta_{A}\left(\left(\tau_{\square}\right)^{*}(x)\right) \text { iff } y \leq \bigwedge\{a \in A \mid x \leq \square a\} \text { iff }(\forall a \in A)(x \leq \square a \Rightarrow y \leq a) .
$$

Thus, $x R_{\zeta_{A} \text { oat }\left(\tau_{\square}\right)} y$ iff $x R_{\square} y$, so $\mathcal{F} \widehat{\mathfrak{a t}} \mathcal{A}(A, \square)=\mathfrak{a t}(A, \square)$, and hence $\mathcal{F} \widehat{\mathfrak{a t}} \mathcal{A}=\mathfrak{a t}$.

Remark 4.13. We conclude the paper by connecting the coalgebraic approaches to JónssonTarski and Thomason dualities. As follows from [KKV04], $\operatorname{Alg}(\mathcal{K})$ is dually equivalent to Coalg $(\mathcal{V})$, from which Jónsson-Tarski duality follows. By Theorem 4.10, $\operatorname{Alg}(\mathcal{H})$ is dually equivalent to $\operatorname{Coalg}(\mathcal{P})$, from which Thomason duality follows.

Let $\mathcal{U}:$ Stone $\rightarrow$ Set be the forgetful functor. For each $X \in$ Stone, viewing the underlying set of the Vietoris space $\mathcal{V}(X)$ as a subset of $\mathcal{P}(X)$, we have an inclusion map $i: \mathcal{U V}(X) \rightarrow \mathcal{P U}(X)$. We extend $\mathcal{U}$ to a forgetful functor on the level of coalgebras. Let $(X, g) \in \operatorname{Coalg}(\mathcal{V})$, so $g: X \rightarrow \mathcal{V}(X)$ is a continuous map. Set $\mathcal{U}(X, g):=\left(\mathcal{U}(X), g^{\prime}\right)$ where $g^{\prime}: \mathcal{U}(X) \rightarrow \mathcal{P U}(X)$ is given by $g^{\prime}=i \circ \mathcal{U}(g)$.

$$
\mathcal{U}(X) \underset{g^{\prime}}{\stackrel{\mathcal{U}(g)}{\longrightarrow} \mathcal{U V}(X) \stackrel{i}{\longrightarrow}} \mathcal{P U}(X)
$$

Then $\left(\mathcal{U}(X), g^{\prime}\right) \in \operatorname{Coalg}(\mathcal{P})$. If $\alpha:\left(X_{1}, g_{1}\right) \rightarrow\left(X_{2}, g_{2}\right)$ is a Coalg $(\mathcal{V})$-morphism, it is straightforward to see that $\mathcal{U}(\alpha):\left(\mathcal{U}\left(X_{1}\right), g_{1}^{\prime}\right) \rightarrow\left(\mathcal{U}\left(X_{2}\right), g_{2}^{\prime}\right)$ is a Coalg $(\mathcal{P})$-morphism. This yields a functor $\mathcal{U}: \operatorname{Coalg}(\mathcal{V}) \rightarrow \operatorname{Coalg}(\mathcal{P})$. If we identify $X$ with $\mathcal{U}(X)$ and $g: X \rightarrow \mathcal{V}(X)$ with $g^{\prime}: \mathcal{U}(X) \rightarrow \wp \mathcal{U}(X)$, then $\mathcal{U}(X, g)=(X, g)$. We have thus forgotten the topological structure of $X$ and the continuity of $g$. This justifies thinking about $\mathcal{U}: \operatorname{Coalg}(\mathcal{V}) \rightarrow \operatorname{Coalg}(\mathcal{P})$ as a forgetful functor.

We next define a functor $(-)^{\sigma}: \operatorname{Alg}(\mathcal{K}) \rightarrow \operatorname{Alg}(\mathcal{H})$ which can be thought of as the canonical extension functor for algebras for $\mathcal{K}$. Use Lemma 4.9 to lift the contravariant functors of Stone duality to $\widehat{\mathfrak{u f}}, \widehat{\mathfrak{c l o p}}$ and those of Tarski duality to $\widehat{\mathfrak{a t}}, \widehat{\wp}$ and $\operatorname{set}(-)^{\sigma}=\widehat{\wp} \circ \mathcal{U} \circ \widehat{\mathfrak{u f f}}$. Thus, $(-)^{\sigma} \circ \widehat{\mathfrak{c l o p}}$ and $\widehat{\wp} \circ \mathcal{U}$ are naturally isomorphic, and hence so are $\mathcal{U} \circ \widehat{\mathfrak{u f}}$ and $\widehat{\mathfrak{a t}} \circ(-)^{\sigma}$.

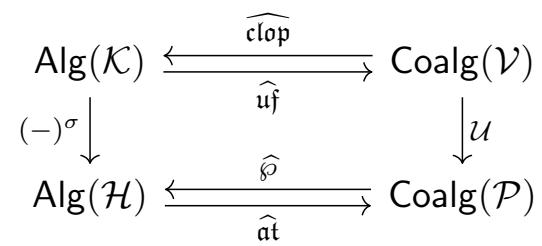

The functor $\mathcal{K}$ satisfies $\mathfrak{u f} \mathcal{K}(A) \cong \mathcal{V} \mathfrak{u f}(A)$ [KKV04, Cor. 3.11]. By identifying these two Stone spaces, for $(A, \alpha) \in \operatorname{Alg}(\mathcal{K})$ we describe $(A, \alpha)^{\sigma}$ directly. Since $\alpha: \mathcal{K}(A) \rightarrow A$ is a BA-morphism, $\mathfrak{u} \mathfrak{f}(\alpha): \mathfrak{u} \mathfrak{f}(A) \rightarrow \mathfrak{u} \mathfrak{f} \mathcal{K}(A)=\mathcal{V} \mathfrak{u f}(A)$ is a continuous map. Therefore, $(\mathfrak{u f}(A), \mathfrak{u f}(\alpha)) \in \operatorname{Coalg}(\mathcal{V})$. The forgetful functor (after appropriate identifications) sends this 
to $(\mathfrak{u} \mathfrak{f}(A), \mathfrak{u f}(\alpha)) \in \operatorname{Coalg}(\mathcal{P})$. Finally, $\widehat{\wp}$ sends $(\mathfrak{u} \mathfrak{f}(A), \mathfrak{u f}(\alpha))$ to $(\wp \mathfrak{u} \mathfrak{f}(A), \wp \mathfrak{u} \mathfrak{f}(\alpha))=\left(A^{\sigma}, \alpha^{\sigma}\right)$. Because of these calculations, we can view the functor $\operatorname{Alg}(\mathcal{K}) \rightarrow \operatorname{Alg}(\mathcal{H})$ as the canonical extension functor for algebras for $\mathcal{K}$.

\section{ACKNowledgements}

We would like to thank the referees for their comments. One of the referees suggested to us an alternative description of $\mathcal{L}$ given in Theorem 3.9. We also thank Nick Bezhanishvili, Jim de Groot, Sebastian Enqvist, Clemens Kupke, Phil Scott, and Yde Venema for their comments on an earlier version of the paper.

\section{REFERENCES}

[Abr88] S. Abramsky. A Cook's tour of the finitary non-well-founded sets. Invited Lecture at BCTCS, 1988. Available at arXiv:1111.7148, 1988.

[AHS06] J. Adámek, H. Herrlich, and G. E. Strecker. Abstract and concrete categories: the joy of cats. Repr. Theory Appl. Categ., (17):1-507, 2006.

[BHJ21] G. Bezhanishvili, J. Harding, and M. Jibladze. Canonical extensions, free completely distributive lattices, and complete retracts. Algebra Universalis, 82(4):Article No. 64, 6 pp., 2021.

[Dwi81] Ph. Dwinger. Structure of completely distributive complete lattices. Indag. Math., 43(4):361-373, 1981.

[Esa74] L. L. Esakia. Topological Kripke models. Soviet Math. Dokl., 15:147-151, 1974.

[Gai64] H. Gaifman. Infinite Boolean polynomials. I. Fund. Math., 54:229-250, 1964.

[GH01] M. Gehrke and J. Harding. Bounded lattice expansions. J. Algebra, 238(1):345-371, 2001.

[Gol76] R. I. Goldblatt. Metamathematics of modal logic. Rep. Math. Logic, (6):41-77, 1976.

[Hal56] P. R. Halmos. Algebraic logic. I. Monadic Boolean algebras. Compositio Math., 12:217-249, 1956.

[Hal64] A. W. Hales. On the non-existence of free complete Boolean algebras. Fund. Math., 54:45-66, 1964.

[HMS74] K. H. Hofmann, M. Mislove, and A. Stralka. The Pontryagin duality of compact O-dimensional semilattices and its applications. Lecture Notes in Mathematics, Vol. 396. Springer-Verlag, BerlinNew York, 1974.

[Jac17] B. Jacobs. Introduction to coalgebra, volume 59 of Cambridge Tracts in Theoretical Computer Science. Cambridge University Press, Cambridge, 2017.

[Joh82] P. T. Johnstone. Stone spaces, volume 3 of Cambridge Studies in Advanced Mathematics. Cambridge University Press, Cambridge, 1982.

[JT51] B. Jónsson and A. Tarski. Boolean algebras with operators. I. Amer. J. Math., 73:891-939, 1951.

[KKV04] C. Kupke, A. Kurz, and Y. Venema. Stone coalgebras. Theoret. Comput. Sci., 327(1-2):109-134, 2004.

[Kri63] S. A. Kripke. Semantical considerations on modal logic. Acta Philos. Fenn., Fasc.:83-94, 1963.

[Mac71] S. Mac Lane. Categories for the working mathematician. Graduate Texts in Mathematics, Vol. 5. Springer-Verlag, New York, 1971.

[Mar79] G. Markowsky. Free completely distributive lattices. Proc. Amer. Math. Soc., 74(2):227-228, 1979.

[MT44] J. C. C. McKinsey and A. Tarski. The algebra of topology. Ann. of Math., 45:141-191, 1944.

[Tay02] P. Taylor. Subspaces in abstract Stone duality. Theory Appl. Categ., 10, 2002.

[Tho75] S. K. Thomason. Categories of frames for modal logic. J. Symb. Log., 40(3):439-442, 1975.

[Ven07] Y. Venema. Algebras and coalgebras. In Handbook of modal logic, volume 3, pages 331-426. Elsevier B. V., Amsterdam, 2007. 